\title{
Deux nouvelles espèces de Lumbriculidae du Sud-Ouest de l'Europe
}

\author{
N. Giani 1 \\ E. Martinez-Ansemil2
}

Mots clés: Oligochaeta, Lumbriculidae, taxinomie, nouvelles espèces.

Rhynchelmis paraolchonensis $\mathrm{n}$. sp. a été récoltée dans un étang des Landes (France). Elle appartient au groupe des Rhynchelmis dont les muscles longitudinaux ne sont pas disposés en 8 masses recourbées et dont la spermathèque n'est pas en communication avec l'cesophage. Cette forme est proche de $\boldsymbol{R}$. olchonensis Burov et Kozov, du lac Baîkal.

Stylodrilus glandulosus n. sp. a été récoltée dans le fleure Mino en Espagne. Elle possède un atrium long, comme $S$. subcarpathicus (Hrabe) et $S$. longiatriatus Dembitsky. Elle se caractérise par son bulbe pénien très développé et par la présence de massifs glandulaires à la base de la spermathèque et au niveau du sac pénien.

Two new species of Lumbriculidae from the South-West of Europe.

Keywords : Oligochaeta, Lumbriculidae, taxonomy, new species.

Rhynchelmis paraolchonensis n. sp. has been collected from a pond near Landes (France). It belongs to the Rhymchelmis group whose longitudinal muscles are not arranged in eight recurved masses and whose spermatheca is not in communication with the oesophagus. This form is close to $R$. olchonensis Burov and Kozov, from lake Baikal.

Stylodrilts glandulosus $\mathrm{n}$. sp. has been collected from the river Mino in Spain. It has a long at rium, as in $S$. subcarpathicus (Hrabe) and S. longia riatus Dembitsky. Its chief characteristics are a well-developed penis bulb and glandular masses at the base of the spermatheca and at the level of the penal sac.

\section{1. - Description de Rhynchelmis paraolchonensis n. sp.}

\section{MORPHOLOGIE EXTERNE}

Longueur (matériel fixé) : $\mathbf{3 5 - 4 5} \mathrm{mm}$. Nombre de segments : 125-192. Le diamètre du corps croît dans la région antérieure jusqu'au niveau des segments génitaux :

$\begin{array}{lcccc}\text { Segment } & \text { II } & \text { V } & \text { VIII } & \text { XI } \\ \text { Diamètre }(\mu \mathrm{m}) & 280 & 340 & 465 & 630\end{array}$

\footnotetext{
1. Laboratoire d'Hydrobiologie, UA 695, Université P, Sabatier, 118, route de Narbonne, 31062 Toulouse Cedex France.

2. Colegio Universitario de Orense, c. General Franco, 35. Orense, Espagne.
}

Il existe une double annulation sur les segments antérieurs à partir du segment II mais bien marquée à partir du segment III seulement. Le prostomium est arrondi à son extrémité (fig. $1 \mathrm{~A}$ ) et il est légèrement plus large $(195-250 \mu \mathrm{m})$. La paroi du corps a une épaisseur de 40 à $45 \mu \mathrm{m}$ au niveau du segment VI et de $60 \mu \mathrm{m}$ au niveau du clitellum. La couche de muscles longitudinaux mesure de 27 à $34 \mu \mathrm{m}$ et les muscles circulaires ne dépassent pas $4 \mu \mathrm{m}$ d'épaisseur. L'épiderme, épais de $12 \mu \mathrm{m}$ en VI atteint 25-26 $\mu \mathrm{m}$ au niveau du clitellum. La cuticule est mince. Sur le matériel fixé le tégument a un aspect irisé comparable à celui de Lumbriculus variegatus (Müller). Le clitellum occupe les segments IX à XIII inclus.

Il y a deux soies à pointe simple dans chaque faisceau. La longueur et l'épaisseur de ces soies croîs- 

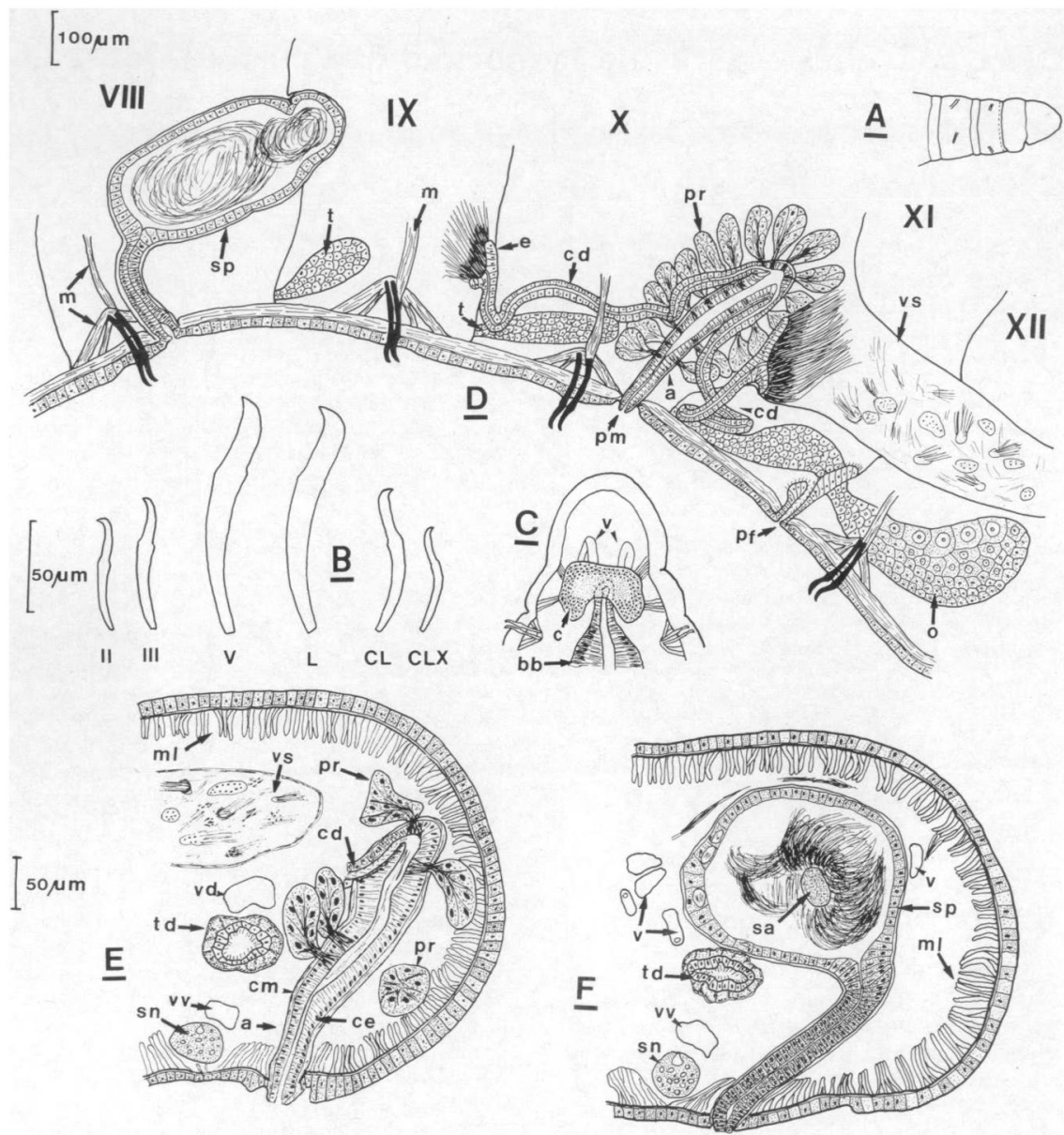

Fig. 1. Rhynchelmis paraolchonensis $\mathbf{n}$. sp. A : prostomium et région antérieure. $\mathbf{B}$ : soies (les chiffres romains corres pondent au $n^{0}$ du segment). $C$ : région antérieure en vue dorsale mont rant le cerveau. $D:$ Mise en place des appareils génitaux (d'après dissection). $E$ : coupe transversale au niveau du segment $X$. $F$ : coupe transversale au niveau du segment VIII.

(a : atrium, bb : bulbe buccal, c : cerveau, cd : canal déférent, ce : couche épithélienne, cm : couche musculaire, e : entonnoir spermatique, $m$ : muscles, $m$ l : muscles longitudinaux, $o$; ovaire, pf : pore femelle, pm : pore mâle, $\mathrm{pr}$ : prostate, sa : sécrétion amorphe, sn : chaîne nerveuse, $s p:$ spermathèque, $t:$ testicule, $t d:$ tube digestif, $v:$ vaisseau sanguin, vd : vaisseau dorsal, vs: vésicule séminale, vv : vaisseau ventral). 
Tableau I. Longueur et épaisseur des soies mesurées sur deux individus.

\begin{tabular}{lcccccccccc}
\hline $\mathrm{N}^{\circ}$ du segment & II & III & IV & V & VII & IX & XI & XX & LV & CVIII \\
\hline $\begin{array}{c}\text { longueur } \\
\text { en } \mu \mathrm{m}\end{array}$ & 56 & 73 & & 117,5 & & 120,5 & 131,5 & 134,5 & 140 & 62 \\
$\begin{array}{c}\text { enaisseur } \\
\text { en } \mu \mathrm{m}\end{array}$ & 5,1 & 6,8 & 112 & & 154 & & 168 & & \\
& 4,2 & & 13,6 & & & 12 & 13,5 & 12 & 13.5 & 6.5 \\
\hline
\end{tabular}

sent progressivement dans la région préclitellienne (fig. 1 B) pour diminuer dans la région postérieure (tableau I) où les soies sont identiques à celles du segment II. I e nodulus est presque médian en II et dans les segments postérieurs : le rapport partie distale/longueur totale de la soie est compris entre 0,4 et 0,45 . Sur les autres segments les soies ont un nodule distal (rapport compris entre 0,3 et 0,35 ).

\section{ANATOMIE}

Le bulbe buccal est allongé et il occupe les segments II, III et le début de IV. Il existe des glandes pharyngiennes à la fin du segment IV mais celles des segments V, VI et VII sont plus développées. Les cellules chloragogènes débutent sur le segment VII et elles forment une couche de 70 à $120 \mu \mathrm{m}$ d'épaisseur autour du tube digestif. Le cerveau, échancré postérieurement, est entièrement compris dans le péristomium. Dans la région antérieure une paire de vaisseaux transversaux très longs unissent les vaisseaux dorsa] et ventral. Au niveau du clitellum ces vaisseaux pénètrent dans les sacs spermatiques et ovocytaires. Dans la région postérieure le vaisseau dorsal rejoint directement le sinus périviscéral sans former de vaisseaux latéraux libres dans la cavité.

Les ovaires sont at tachés à la partie inférieure du dissépiment $\mathbf{X} / \mathbf{X I}$ et ils se développent en XI et XII (fig. $1 \mathrm{D})$. Les ovocytes en cours de maturation sont localisés en bordure de la partie postérieure de l'ovaire. Le sac ovocytaire postérieur peut atteindre les segments XIX ou XX. Le tractus génital femelle (fig. 1 D) est formé $\mathrm{d}$ 'un pavillon cilié bien développé (hauteur : $70 \mu \mathrm{m}$ ) situé dans le segment XI sur la face antérieure du dissépiment XI/XII. Le pore femelle est situé dans l'intersegment XI/XII.

Il existe deux paires de testicules localisés en IX et $\mathrm{X}$ et attachés aux dissépiments VIII/IX et IX/X. Le sac spermatique an térieur est peu développé; le postérieur peut at teindre le segment XVII.

L'atrium est situé dans le segment $X$. Le tractus génital mâle comprend deux paires d'entonnoirs spermatiques situés sur les dissépiments IX/X et $\mathrm{X} / \mathrm{XI}$; les postérieurs sont plus développés que les antérieurs et ils s'ouvrent dans les sacs spermati. ques postérieurs (fig. $1 \mathrm{D}$ ). Les canaux déférents ont un diamèt re compris entre 17 et $20 \mu \mathrm{m}$; leur lumière interne est ciliée et a un diamètre de 4 à $7 \mu \mathrm{m}$. Ces canaux sont contournés et le postérieur pénètre dans la partie antérieure du segment XI où il effectue quelques boucles. Ces canaux remontent le long de l'atrium dans lequel ils penètrent subapicalement.

L'atrium est formé d'une ampoule piriforme allongée d'environ 300 à $360 \mu \mathrm{m}$ de longueur et de diamètre compris entre 60 et $65 \mu \mathrm{m}$. Parfois les atria pénètrent dans les sacs spermatiques jusque dans la partie antérieure du segment XI. L'atrium se prolonge par un pénis très court situé dans un sac pénien réduit. Les pores mâles s'ouvrent en arrière des soies du segment $\mathbf{X}$ près de la ligne médiane du corps.

L'atrium est couvert de cellules prostatiques regroupées en amas distincts de forme allongée ou piriformes (100 à $125 \mu \mathrm{m}$ de longueur). Leur longueur est supérieure au diamètre de l'atrium. Ces amas se déversent séparément dans l'atrium par un canal court qui leur donne un aspect pédonculé.

Les spermathèques sont situées dans le segment VIII ; l'une est dirigée vers l'avant, l'autre vers l'arrière. Les pores des spermathèques sont situés en arrière du plan transversal des soles du segment VIII près de la ligne médiane du corps. Chaque spermathèque est constituee d'une ampoule et d'un canal bien distinct. L'ampoule est longue ( $530 \mu \mathrm{m}$ environ) et son diamètre est d'environ $200 \mu \mathrm{m}$; la paroi, épaisse de 5 à $8 \mu \mathrm{m}$ comporte des vacuoles de résorption. Le canal est long de 210 à $225 \mu \mathrm{m}$ et son diamètre varie entre 35 et $42 \mu \mathrm{m}$. Il est formé d'une couche musculaire externe de $3,5 \mu \mathrm{m}$ environ et d'une couche épithélienne très épaisse : la lumière interne est très petite $(1,5 \mu \mathrm{m}$ environ) et elle présente une légère dilatation à la base du canal (fig. 1 F). Dans les ampoules les spermatozoides sont rangées en 1 ou 2 masses compactes entourant des résidus amorphes. 
Tableau II. Comparaison des principaux caractères distinctifs entre notre forme, $R$. olchonensis, $R$. Orientalis et $R$. alaskana.

\begin{tabular}{|c|c|c|c|c|}
\hline & Notre forme & R. olchonensis & R. orientalis & R. alaskana \\
\hline
\end{tabular}

\section{MATERIEL EXAMINE}

9 individus récoltés le 15/09/1977 dans des amas de feuilles mortes dans l'étang Hardy (département des Landes, France). 5 individus ont été disséqués et montés au baume du canada ; 4 ont été coupés au microtome (1 transversalement et 3 longitudinalement).

Discussion

La présence d'une paire de spermathèques sur le segment VIII, d'une paire d'atria en $X$ et de deux paires de testicules (l'une en IX, l'autre en X) sont autant de caractères permettant de ranger cette forme dans le genre Rhynchelmis Hoffmeister.

La division des muscles longitudinaux en 8 masses recourbées latéralement et l'existence d'une communication entre la spermathèque et le tube digestif sont également des caractères distinctifs du genre Rhynchelmis. Notre forme ne présente aucun de ces deux caractères. Mais, $R$. olchonensis Burov et Kozov, 1932, $R$. tetratheca Michaelsen, 1920, $R$. orientalis Yamaguchi, 1936 et $R$. alaskana Holmquist, 1976 ne présentent pas non plus ce type de musculature. De mêrne $R$. orientalis, $R$. olchonensis et $R$, alaskana sont des espèces dépourvues de connection entre la spermathèque et le tube digestif.

Le tableau II permet de comparer rapidement notre forme aux trois dernières espèces citées. Il apparaît que nos spécimens sont très proches de $R$. olchonensis dont ils diffèrent par le nombre de seg. ments, la position du clitellum, la longueur du canal déférent postérieur... Ces différences, associées à la localisation géographique de $R$. olchonensis (Lac Baïkal) nous conduisent à établir une nouvelle espèce : $\boldsymbol{R}$. paraolchonensis n. sp. du Sud-Ouest de la France.

Hrabe (1982) a établi le genre Pseudorhynchelmis pour $R$. olchonensis. Parmi tous les caractères invoqués pour définir ce genre, un seul semble être exclusif de cette espèce : la longueur du canal déférent postérieur est supérieure à celle de l'atrium.

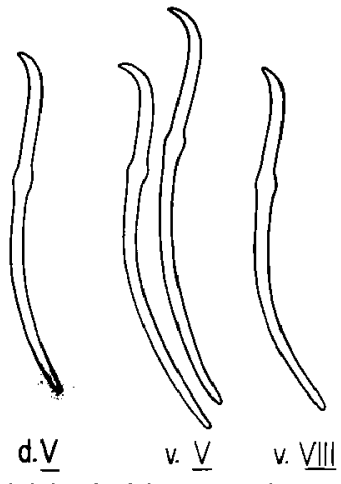

Fig. 2. Stylodrilus glandulosus n. sp. : dV : soies dorsales du segment $V$; $v$. $V$; soies vent rales du segment $V$; v. VIII : soies ventrales du segment VIII.

Tous les autres caractères invoqués se retrouvent chez d'autres espèces de Rhynchelmis et la création du genre Pseudorhynchelmis ne nous semble pas justifiée. De même, Holmquist (1976) ne trouve aucune raison pour subdiviser le genre Rhynchelmis et elle rejette ainsi le genre Rhynchelmoides Hrabe, 1936.

\section{2. - Description de Stylodrilus glandulosus n. sp.}

\section{CARACTERES EXTERNES}

Longueur (matériel fixé) : $23 \mathrm{~mm}$ ( 1 individu). Deux individus entiers possédaient respectivement 68 et 76 segments. Le diamètre du corps, mesuré au niveau du segment VI, est d'environ $370 \mu \mathrm{m}$. La double annulation apparaît au niveau du segment IV et elle est très marquée à partir du segment $V$. Le prostonium est généralement un peu plus long (211 à $290 \mu \mathrm{m}$ ) que large (245 à $283 \mu$ ) et il est arrondi à son extrémité. 
Le clitellum est peu élevé et il occupe les segments IX, X, XI, XII et XIII. Parfois il débute seulement au niveau des soies du segment IX. L'épiderme a une épaisseur de 7 à $10 \mu \mathrm{m}$ sauf dans la région clitellienne où il est d'environ 13 à $18 \mu \mathrm{m}$. Une paire de pores mâles sont localisés dans une dépression ven. trale de la paroi du corps sur le segment $X$. Une paire de pores des spermathèques sont présents en IX.

Chaque segment possède 4 faisceaux de 2 soies. Il existe de nombreuses soies de remplacement et de nombreux faisceaux comportent 4 soies; sur un individu, nous avons même noté au niveau des soies latéro-ventrales du segment $\mathrm{X}$ un faisceau comportant 7 soies. Il s'agit de soies sigmoïdes à pointe simple dont la courbure distale est plus ou moins pro. noncée (fig. 2) sans qu'il y ait de relation avec la position de la soie sur le corps de l'animal. Les soies du segment II sont plus courtes et plus fines que celles des segments suivants et les soies dorsales sont toujours un peu plus courtes mais de la même épaisseur que les ventrales.

\section{Soies dorsales Soies ventrales} longueur : longueur : épaisseur : Segment II : $\quad 82-84 \mu \mathrm{m} \quad 106-132 \mu \mathrm{m} \mathrm{4.3.4,8} \mu \mathrm{m}$ Autres segments $\quad 103-117 \mu \mathrm{m} \quad 125-159 \mu \mathrm{m} \mathrm{5,3.5,9} \mu \mathrm{m}$

\section{CARACTERES INTERNES}

Le cerveau est petit, ovoïde et entièrement contenu dans le péristomium (longueur : 105 et $106 \mu \mathrm{m}$; diamètre : 79 et $84 \mu \mathrm{m}$. Le pharynx occupe les segments II, III et une partic de IV. Des glandes pharyngiennes sont présentes en IV, V, VI et VII. Les plus développés sont celles de V. Celles de VII sont les plus réduites et elles sont limitées à la partie antérieure du segment. En VI et VII, les glandes pharyngiennes sont localisées à la partie inférieure du tube digestif alors que celles de IV et $\mathrm{V}$ sont également bien développées dans la région supérieure. Les cellules chloragogènes débutent au niveau du segment VI. L'appareil circulatoire n'a pu être observé correctement compte tenu de la nature du fixateur utilisé. Cependant, nous avons pu noter la présence d'anses non renflées en cœur au niveau des segments II, III, IV et V. Il en existe probablement au niveau des autres segments préclitelliens. Dans les segments postérieurs, le vaisseau dorsal èmet deux expansions aveugles, difficiles à voir car elles sont englobées dans les cellules chloragogènes comme le vaisseau dorsal lui-même. Nous les avons cependant bien observées sur un individu au niveau des segments XXXVIII à XL.

Appareil excréteur : le $1^{\circ}$ pore néphridien s'ouvre sur le segment VII à proximité et en avant des soies

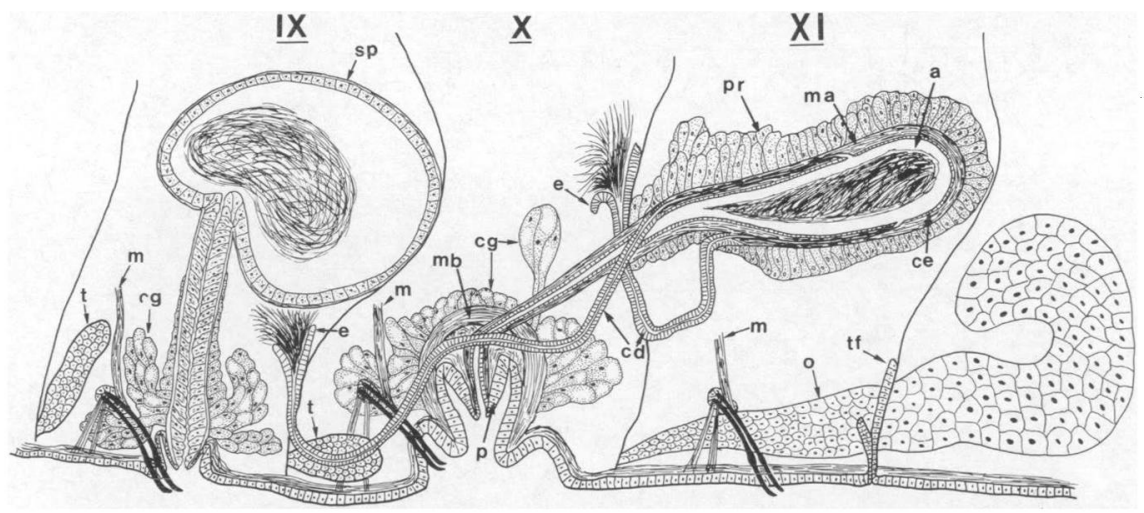

Fig. 3. Stylodrilus glandulosus n. sp. Structure de l'appareil génital (d'après dissection). cg : cellules glandulaires ; ma : muscles de l'atrium ; mb : muscles du bulbe pénien ; $p$ pénis ; tf : tractus génital femelle ; pour les autres lettres, voir figure 1 


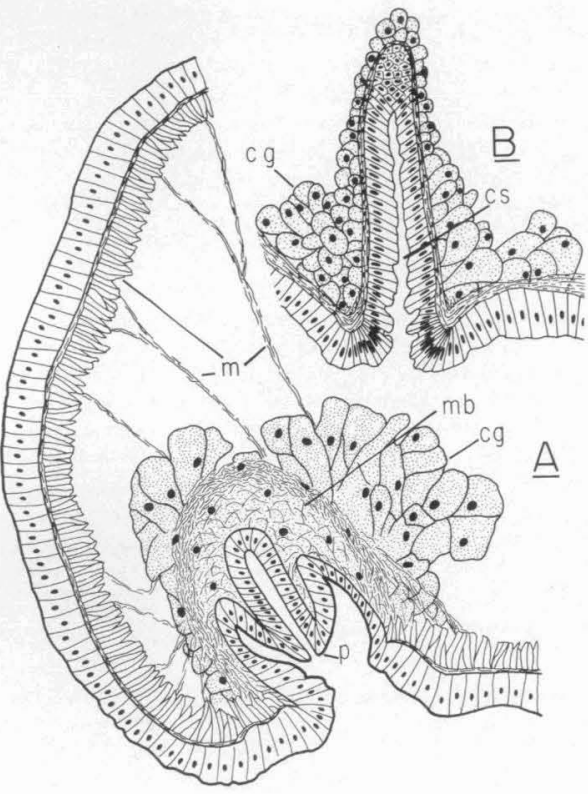

Fig. 4. Stylodrilus glandulosus $\mathrm{n}$. $\mathrm{sp}$. A : coupe transversale au niveau du pore màle. B : coupe transversale au niveau du pore de la spermathèque cs : canal spermathècal; pour les aut res lettres voir figures 1 et 3 .

Fig. 5. Sivlodrilus glandulusus $\mathrm{n}$. $\mathrm{sp}$ : coupe transversale au niveau de l'atrium (legende: voir figures précédentes).

Fig. 6. Stylodrilus glandulosus n. sp. : coupe transversale au niveau du segment XII. cc : cellules chloragogénes ; $\mathbf{c n}$ : chaîne nerveuse ; muscles; $\boldsymbol{n}$ : néphridies : Id : tube digestif ; vd : vaisseau dorsal ; vs : vésicule séminale; $\mathrm{vw}$ : vaisseau ventral.
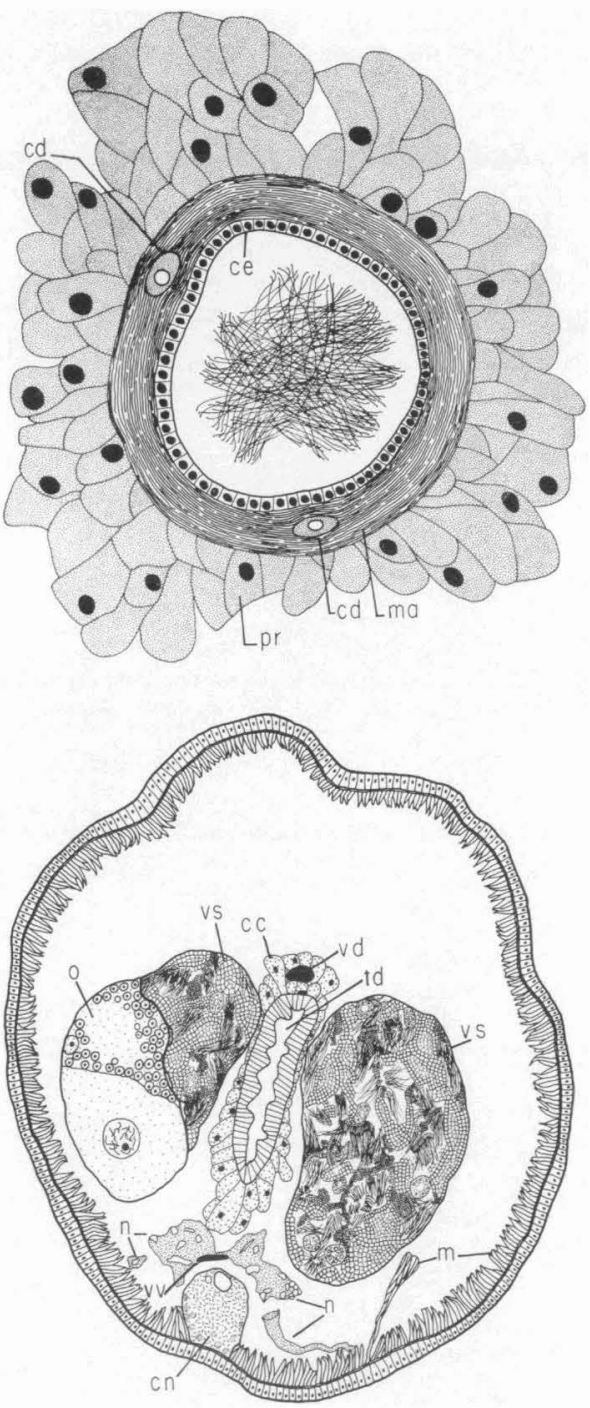
latéro-ventrales. La $1^{\circ}$ paire de néphridies est très longue et elle at teint le segment IX. Il n'y a pas de néphridies sur les segments VIII, IX, X, XI et XII. A partir de XIII, il y a une paire de néphridies par segment dont le pore est situé dans la même posi. tion que celui du segment VII.

Au pavillon cilié fait suite un renflement (glandulaire ?). Les tubules néphridiens viennent ensuite $s^{*}$ accoler au vaisseau sanguin ventral et à la chaîne nerveuse. Les néphridies d'un même segment sont interconnectées et elles sont également connectées à celles des segments suivants. Leur position cor. respond è celle décrite pour Lumbriculus variegatus, Stylodrilus heringianus, Bythonomus absoloni et Trichodrilus allobrogum. Leur structure est très proche de celle de Lumbriculus variegatus telle qu'elle a été figurée par Stephenson (1930).

Appareil génital mâle : une paire de testicules disposés ventralement dans chacun des segments IX et $X$. Ils sont attachés respectivement aux dissépiments VIII/IX et IX/X. Une vésicule séminale se développe à partir des septum VIII/IX et son exten. sion est variable. Elle peut atteindre le segment $V$ et même le segment IV.

La deuxième vésicule séminale se forme à partir du septum X/XI et elle atteint le segment XIV et parfois même le segment XV. Les pores mâles sont situés sur le segment $X$.

Les atria sont allongés : 460 à $660 \mu \mathrm{m}$; ils pénètrent généralement dans le segment XI et dans un cas le segment XII. Sur un individu, un des atria était dirigé vers l'arrière et pénétrait dans le seg. ment XI alors que l'autre était dirigé vers l'avant et restait localisé dans le segment $X$.

L'atrium est constitué d'une ampoule terminale de 120 à $151 \mu \mathrm{m}$ de diamètre maximum. La paroi de cet atrium a une épaisseur de 9 à $13,3 \mu \mathrm{m}$. Elle est formée d'une mince couche de cellules épithéliales entourée par une couche plus épaisse de muscles circulaires. Cette ampoule est couverte de cellules prostatiques hautes. Elle se rét récit en un canal de 42 à $45 \mu \mathrm{m}$ de diamètre environ qui est dépourvu de cellules prostatiques mais couvert de cellules basses, probablement péritonéales. Ce canal se termine par un pénis dont le canal a un diamètre de $26,5 \mu$ pour une lumière interne de 2,5-3 $\mu \mathrm{m}$. Ce canal est entouré d'une couche de muscles circulaires.

Le pénis lui-mème est conique et a une hauteur de 84 à $92 \mu \mathrm{m}$ pour un diamètre basal d'environ 80 $\mu \mathrm{m}$; ce pénis est situé dans un sac pénien entouré d'un grand bulbe pénien de nature musculaire situé juste en arrière des soies du segment $X$. Ce bulbe a une hauteur variant de 100 a $200 \mu \mathrm{m}$ pour un diamètre de 92 à $180 \mu \mathrm{m}$ selon le degré de contraction. Il est formé de fibres musculaires concentriques attachées à la paroi ventrale du corps. Il est entouré par un massif de cellules glandulaires piriformes, assez semblables aux cellules prostatiques $(f \mathrm{ig} .3 \mathrm{et}$ 4 A). Le pénis est également relié à la paroi supérieure du corps par des bandes musculaires ( $f$ ig. 4 A). Le bulbe pénien musculeux et ces bandes musculaires laissent présager que le pénis est à la fois rétractile et dévaginable.

Les deux canaux déférents rejoignent chaque atrium basalement et cheminent dans la musculature atricale pour s'ouvrir subapicalement au niveau de l'ampoule (fig. 3 et 5). L'un de ces canaux est court et il s'ouvre au niveau de l'entonnoir spermatique situé sur le segment X/XI ; l'autre, plus long. parcourt ventralement le segment $X$ puis remonte le long du dissépiment IX/X pour s'ouvrir au niveau d'un entonnoir spermatique dans le segment IX. Ces canaux ont un diamètre de $17 \mu \mathrm{m}$ environ pour une lumière interne d'environ $5 \mu \mathrm{m}$; lorsqu'ils parcourent la paroi musculaire de l'atrium, cette lumière est de 3-4 $\mu \mathrm{m}$ et le diamètre total du canal est de 10 $\mu \mathrm{m}$ maximum et de $6 \mu \mathrm{m}$ minimum.

Appareil génital femelle : il existe une paire d'ovaires attachés basalement au niveau du septum $X / X I$. Ils sont très allongés et occupent toute la longueur du segment XI et du segment XII. Le sac ovocytaire se forme par invagination du septum XI/XII et il peut atteindre les segments XIII ou XIV selon les cas. Le tractus génital femelle est formé de deux pavillons ciliés situés en arrière du segment XI contre le dissépiment XI-XII (fig. 3).

Spermathèque : il existe une paire de spermathèques situées entièrement dans le segment IX. Les pores s'ouvrent sur la ligne des soies ventrales en arrière de celles du segment IX. Chaque spermathèque a une hau teur totale de 400 à $567 \mu \mathrm{m}$; elle est formée d'une ampoule ovoïde (longueur : 186 à $232 \mu \mathrm{m}$; diamètre : 159 à $190 \mu \mathrm{m}$ ) à paroi mince (8 à $9,3 \mu \mathrm{m}$ d'épaisseur). Cette ampoule surmonte un canal distinct de 195 à $355 \mu \mathrm{m}$ de longueur pour un diamètre de 38 à $43 \mu \mathrm{m}$. Ce canal est entouré d'une fine couche de muscles circulaires alors que l'épithélium est très développé et la lumière du canal interne est très faible. 
Ce canal est légèrement renflé près de sa base (le diamètre atteignant jusqu'à $5 ! \mu \mathrm{m}$ ) et il se rétrécit à nouveau avant le pore qui s'ouvre dans une faible invagination tégumentaire en fente transversale.

Le canal de la spermathèque est recouvert extérieurement de cellules glandulaires dont la taille croît progressivement vers la partie basale. La base de ce canal est elle-même entourée d'un massif très développé de cellules glandulaires plus hautes, comparables à celles de l'atrium. Il est vraissemblable que ces cellules qui entourent la base du canal de la spermathèque et le bulbe pénien soient assimilables à des glandes copulatrices. Mais nous n'avons pas pu observer de relation directe entre ces glandes et le canal de la spermathèque ou le pénis.

Les spermatozoïdes sont agglomérés en une masse indistincte au centre de l'ampoulę de la spermathèque (comme dans celle de l'atrium). Dans l'ampoule de la spermathèque on note également la présence d'une sécrétion amorphe dont nous ne pouvons préciser la nature (peut-être résulte-t-elle simplement de la fixation?).

\section{Matériel examiné}

- 4 individus disséqués, colorés à l'hématoxyline et montés au baume du Canada.

-1 individu coupé transversalement au microtome (coupes de $10 \mu \mathrm{m}$ d'épaisseur).

-2 individus coupés longitudinalement au microtome (coupes de $7,5 \mu \mathrm{m}$ ).

Les coupes ont été colorées à l'hématoxylineéosine. Ces 7 spécimens sont conservés dans la collection des auteurs.

\section{LIEUX ET DATES DES RECCOLTES}

Tous les individus ont été capturés le 20/XI/81 dans le fleuve Mino à Orense, Espagne, dans une station située à environ $150 \mathrm{~m}$ en aval d'un lac de barrage (altitude : $130 \mathrm{~m}$; latitude : $\mathrm{N} 42^{\circ} 20^{\prime} 50^{\prime \prime}$, longitude : W $\left.07^{\circ} 51^{\prime} 00^{\prime \prime}\right)$.

\section{Discussion}

La position et la structure de l'appareil génital mâle ( 1 seule paire de spermathèque en IX, atria en $\mathrm{X}$ et la présence de 2 paires de testicules, 1 paire d'ovaires, 2 canaux déférents par atrium) permet- tent de ranger cette forme dans le genre Stylodri. lus sensu Brinkhurst (1965). Dans ce genre, elle se range dans le groupe des espèces à soies à pointe simple et à atrium allongé. Stylod rilus subca rpathicus (sub Bythonomus subcarpathicus) est resté long. temps la seule espèce du genre dont l'atrium très allongé pénétrait dans les segments suivants. Brinkhurst \& Jamieson (1971) avaient même émis des doutes sur l'appartenance de cette espèce au genre Stylodrilus indiquant son appartenance possible au genre Eclipidrilus. Dembitsky (1975) a récolté, en Crimée, une nouvelle espèce Stylodrilus longiatriatus dont la longueur de l'atrium est comparable à celle de Stylodrilus subcarpathicus. L'espèce décrite dans ce travail vient donc se joindre aux deux précédentes. Elle en diffère essentiellement par l'importance et la structure de son bulbe pénien ainsi que par la présence de massifs de cellules glandulaires que nous assimilons à des glandes copulatrices situées à la base de la spermathèque et au niveau du pénis. Ce caractère est d'ailleurs unique dans le genre Stylodrilus. Mais il rapproche cet te espèce des deux genres de Lumbriculidae à atrium allongé : la structure du pénis est voisine de celle des Eclipidri. lus et la présence de glandes copulatrices à la base du pénis (mais pas de la spermathèque) a déjà été observée chez les Lamprodrilus. Brinkhurst (1965) a réuni les anciens genres Stylodrilus et Bythonomus. Hrabe (1970) s'est élevé vivement contre cet te opinion. Sokolskaya (1975) a admis l'opinion de Brinkhurst (1965). La présente espèce à atrium allongé, à soies à pointes simples mais dont les canaux déférents pénètrent apicalement contribue à invalider le genre Bythonomus.

Le pénis rappelle beaucoup celui de Stylodrilus brachystylus.

Travaux cltes

Brinkhurst (R.O.). 1965. - A revision of the genera Stylodrilus and Bythonomus (Oligochaeta, Lumbriculidae). Proc. Zool. Soc. Lond., $144: 431444$

Brinkhurst (R.O.) et Jamieson (B.G.M.). 1971. - Aquatic Otigo. chaeta of the World. Oliver and Boyd, Edinburgh, 860 p.

Dembitzy (E.B.). 1975. - A new species of the gemus Stylodrilus (Oligochaeta) Lumbriculidae from the mountains of Crimea. Zool. Zh. 54:1723-1726.

Holmquist (C.) 1976 . - Lumbriculids (Oligochaeta) of Northern Alaska and Northwestem Canada. Zool. Jb. Syst., 103 S: 377431 . 
Hrabe (S.), 1970. - Notes on the genera Stylodrilus and Bythonomus (Lumbriculidae, Oligochaeta). Spisy Prir. fak. Univ. Purkyne, Bmo, 515 : 283-309.

Hrabe (S.). 1982. - Contribution to the knowledge of Oligochaeta from the Lake Baikal. Vest. Csl. Spol. Zool., 46: 174193.
Sokolskaya (N.L.). 1975. - Une nouvelle espece de Stylodrilus (Oligochaeta, Lumbriculidae) de la presqu"ile Tchoukosky. Zool. Zh., $54: 116-119$.

Stephenson (J.). 1930. - The Oligochaeta. Oxford University Press, $978 \mathrm{p}$. 